\title{
TRATAMENTO CONSERVADOR DE FRATURA CONDILAR ALTA: RELATO DE CASO
}

\author{
CONSERVATIVE TREATMENT OF HIGH CONDYLAR FRACTURE: CASE REPORT
}

\author{
Cristovão Marcondes de Castro RODRIGUES'; Daniela Meneses SANTOS'; Mirlany Mendes Maciel OLIVEIRA ${ }^{1}$; Marcelo Caetano \\ Parreira SILVA2; Lair Mambrini FURTADO ${ }^{3}$ \\ 1 - Residente de Cirurgia e Traumatologia Bucomaxilofacial, Hospital de Clínicas da Universidade Federal de Uberlândia (HC-UFU), Uberlândia, \\ Minas Gerais, Brasil. \\ 2 - Preceptor da Residência de Cirurgia e Traumatologia Bucomaxilofacial, Hospital de Clínicas da Universidade Federal de Uberlândia (HC-UFU), \\ Uberlândia, Minas Gerais, Brasil. \\ 3 - Doutor em Patologia pela Universidade Federal do Triângulo Mineiro (UFTM), Preceptor da Residência de Cirurgia e Traumatologia \\ Bucomaxilofacial, Hospital de Clínicas da Universidade Federal de Uberlândia (HC-UFU), Uberlândia, Minas Gerais, Brasil.
}

\section{RESUMO}

Objetivo: Este trabalho tem como objetivo descrever um caso clínico de paciente com fratura côndilar tratado de forma incruenta com acompanhamento de dezoito meses. Relato de caso: paciente jovem, do sexo masculino, vítima de acidente motociclístico evoluindo com mordida aberta anterior, limitação da abertura bucal, dor bilateralmente nas articulações temporo-mandibulares, ausência de excursão dos côndilos em abertura, limitação dos movimentos de lateralidade e protrusão mandibular. Foram realizados exames de imagem e diagnosticado com fratura bilateral de côndilo alta. A conduta adotada foi a realização de instalação de barra de erich bimaxilar e bloqueio maxilo-mandibular por 21 dias, posterior instalação de elásticos para guiar oclusão, fisioterapia intensiva e acompanhamento ambulatorial mensalmente. Considerações finais: mesmo com as distintas vertentes literárias quanto ao tratamento das fraturas côndilares, o tratamento fechado mostra-se eficaz, sendo de consenso que o diagnóstico precoce, intervenção imediata e retorno extemporâneo das atividades funcionais, visando promover um melhor prognóstico no tratamento de fraturas.

Palavras-chave: Côndilo Mandibular; Articulação Temporo-mandibular; Dimensão Vertical; Tratamento Conservador.

\section{INTRODUÇÃO}

As fraturas faciais apresentam importância devido às suas consequências físicas, emocionais e sócio-econômicas ${ }^{1,2}$. Devido a posição ocupada pela mandíbula na face, os traumas atingem essa região com frequência, levando alterações anatômicas e estéticas na face bem como alterar funções básicas necessárias, como mastigação, fonação e deglutição ${ }^{3,4}$.

A etiologia é variada e a gravidade da fratura depende da intensidade e direção do trauma. As quedas acidentais, acidentes automobilísticos, motociclísticos e agressões físicas são os principais fatores etiológicos associados às fraturas condilares ${ }^{5}$.

O côndilo mandibular é o local de menor resistência da mandíbula, e tanto traumas diretos como os indiretos, podem causar a sua fratura, devido a transmissão das forças de impacto ao longo do arco mandibular principalmente em região mentual ${ }^{3,6}$.

Os sinais clínicos característicos de fratura côndilar são: assimetria facial, edema pré-auricular, mordida aberta anterior e ausência de excursões e protrusão da mandíbula, dor, trismo e desvio da linha média dentária mandibular em direção ao lado fraturado ${ }^{4,5}$. As fraturas de côndilo são classificadas de diversas maneiras descritas na literatura, baseando-se na localização anatômica da fratura, quantidade de pontos de fratura e nas estruturas anatômicas envolvidas ${ }^{2,3}$.
Spiessl e Schroll ${ }^{7}$ (1972) estabeleceram uma classificação para fraturas côndilares assim distribuída: Tipo I (fraturas côndilares sem deslocamento), Tipo II (fraturas baixas com deslocamento), Tipo III (fraturas altas com deslocamento), Tipo IV (fraturas baixas com deslocamento da cabeça do côndilo para fora da cavidade glenoidea), Tipo V (fraturas altas com deslocamento da cabeça do côndilo para fora da cavidade glenoidea) e Tipo VI (fraturas intracapsulares). Outra forma de classificação deve considerar o nível em que ocorre a fratura, podendo ser denominadas fraturas altas, médias e baixas ${ }^{1,4}$.

Nenhuma outra fratura na região maxilofacial tem tanta controvérsia na literatura a respeito da escolha terapêutica, uma vez que diversos fatores devem ser observados, tais como: idade, localização e tipo da fratura, grau de deslocamento do segmento fraturado, presença de outras fraturas faciais associadas, presença de dentes, possibilidade de restabelecimento da oclusão dentária e o tempo transcorrido do trauma influenciam na escolha pela abordagem cirúrgica ou conservadora ${ }^{1,7}$.

Esse trabalho tem como finalidade relatar um caso de fratura côndilar alta em que foi adotada conduta conservadora, com acompanhamento de 18 meses e evolução satisfatória. 


\section{RELATO DE CASO}

O paciente participante assinou o termo de consentimento livre e esclarecido, autorizando o uso e a divulgação de suas informações e imagens para fins científicos.

Paciente G.R.C., de 16 anos de idade, sexo masculino, leucoderma, deu entrada no Pronto-Socorro Hospital de Clínicas da Universidade Federal de Uberlândia, vítima de atropelamento, evoluindo com fratura de tíbia e dores em região pré-auricular. Ao exame clínico notou-se edema em região pré-auricular bilateral, quadro de limitação de abertura bucal $(11 \mathrm{~mm})$, dor pré-auricular, ausência de excursão dos côndilos, mordida aberta anterior, contato oclusal posterior deflexivo bilateral, ausência de perdas dentarias. Estabeleceu-se como hipótese de diagnóstico; fratura bilateral de côndilos. Foi solicitado exame de tomografia computadorizada para melhor avaliação e decisão de conduta, onde se observou fratura côndilar alta (intracapsular) bilateral (Figura-1).

Após discussão com equipe, foi decidido pela eleição do tratamento conservador. Em seguida o paciente foi submetido à instalação de barra de Erich superior e inferior sob anestesia local e bloqueio maxilo-mandibular fio de aço, por dez dias, prescrição domiciliar com dexametasona $4 \mathrm{mg}$ uma vez ao dia por três dias, dipirona sódica 500mg 35 gotas 3x ao dia e compressa de água morna no locar de dor.

Após sete dias, paciente retornou ao ambulatório, para reavaliação. O mesmo referiu melhora álgico pré-auricular e notado regressão do edema. O bloqueio maxilo-mandibular com fios de aço foi removido e paciente submetido a exercícios para

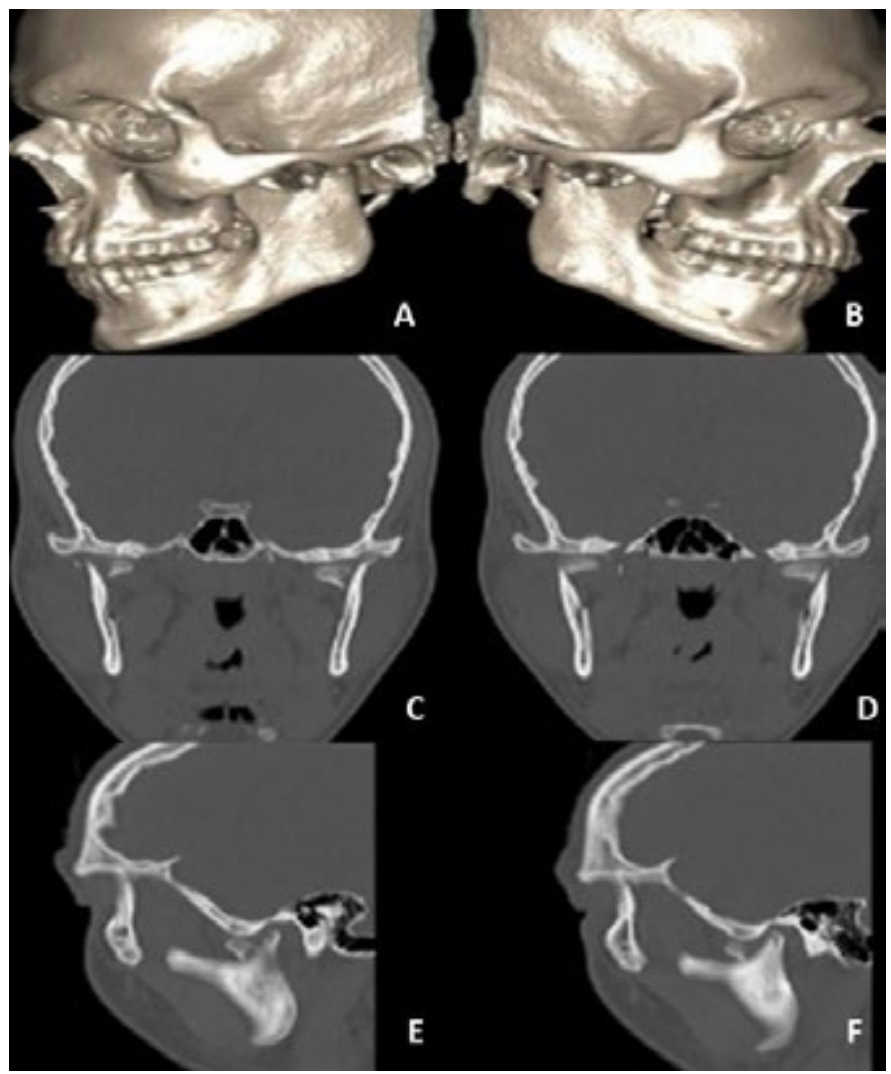

Figura 1 - Tomografia Computadorizada após admissão no serviço CTBMF. Reconstrução 3D do lado esquerdo (A) e lado direito (B). Cortes coronais mostrando a fratura alta de côndilo bilateral deslocada para medial (C e D). Cortes sagital do lado direito e esquerdo mostrando a fratura em nível intracapsular (E - F). avaliação oclusal. Posteriormente à avaliação, constatou-se persistência de alteração oclusal com mordida aberta anterior e mordida cruzada posterior esquerda. Foi decidido colocação de quatro elásticos médios com finalidade de guiar chave de oclusão em classe I de caninos e molares. O paciente foi orientado retornar a cada 72 horas para reavaliação de oclusão troca de elásticos e dieta líquida pastosa.

Após um mês de acompanhamento o paciente retornou ao ambulatório para troca de elásticos e início de fisioterapia com espátulas para estabelecer mobilização articular precoce. Antes de iniciar fisioterapia, encontrava-se com abertura bucal de 20 $\mathrm{mm}$, após sessão de fisioterapia paciente com abertura bucal imediata de $33 \mathrm{~mm}$. O mesmo foi orientado a realizar fisioterapia domiciliar três vezes ao dia, compressas de água morna e retorno ambulatorial três vezes por semana. Foram mantidos quatro elásticos médios para guiar chave de canino classe I.

No retorno de três meses, o paciente compareceu ao ambulatório para realização de fisioterapia e troca de elásticos, com abertura bucal de $36 \mathrm{~mm}$ antes da realização da sessão de fisioterapia e após a fisioterapia com espátulas, a abertura bucal imediata foi de $42 \mathrm{~mm}$ de abertura; mantidas as orientações de compressa de água morna e dieta restritiva (líquida pastosa).

No retorno ambulatorial de seis meses, após o trauma o paciente apresentou uma oclusão satisfatória, abertura bucal antes da realização da fisioterapia, sem queixas álgicas. A barra de Erich foi removida a dieta foi liberada e o paciente orientado retornar ao ambulatório mensalmente e manter realização da fisioterapia uma vez ao dia.

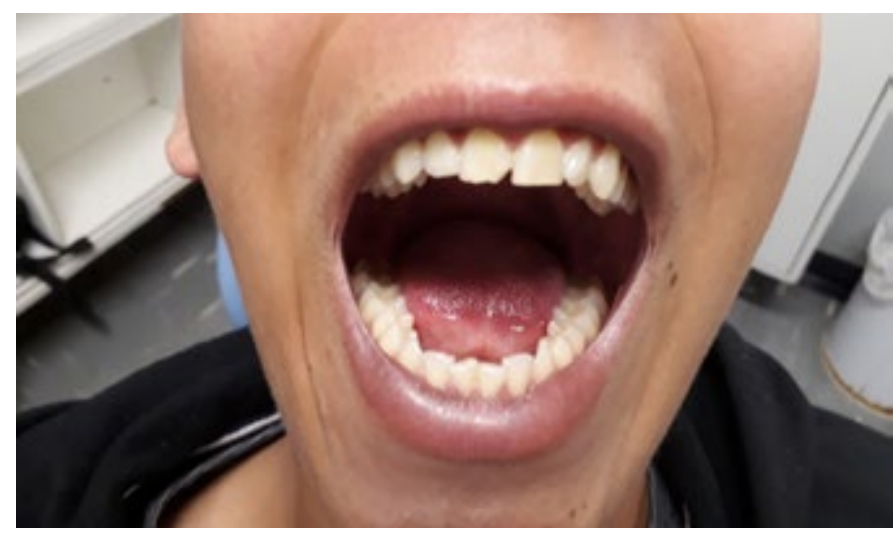

Figura 2 - Abertura Bucal satisfatória após 6 meses do trauma.

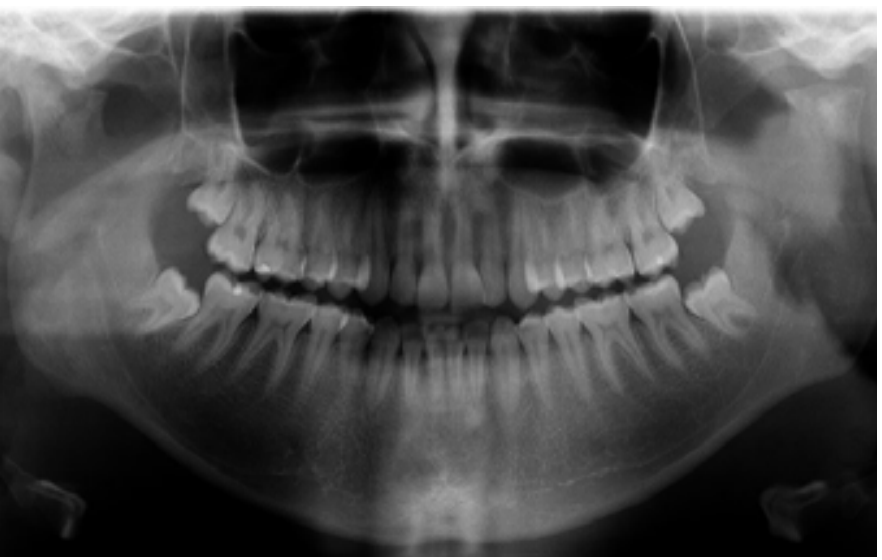

Figura 3 - Radiografia Panorâmica de acompanhamento de 18 meses. 
Após dezoito meses decorridos do trauma, o paciente manteve-se sem queixas, sem alterações oclusais e com abertura bucal satisfatória (Figura-2).

Na radiografia panorâmica de controle observou-se remodelação côndilar satisfatória (Figura-3). Paciente mantem retornos ambulatoriais semestrais.

\section{DISCUSSÃO}

Dentre as diversas fraturas que acometem esse osso gnático, as fraturas côndilares têm se colocado como umas das mais comuns e desafiadoras lesões a serem tratadas ${ }^{1,5}$.

Diante da suspeita de fratura, o profissional deve utilizar métodos complementares para fechamento do diagnóstico, dentre eles, destaca-se os exames de imagem. O padrão ouro para a traumatologia é a tomografia computadorizada com reconstrução, que permite de maneira segura uma análise quantitativa e qualitativa, livre de distorções. Outra opção seria a utilização de exames radiográficos que provêm uma boa visualização local com menor custo. Os mais utilizados são a incidência de Towne, radiografia panorâmica e a incidência lateral oblíqua de mandíbula ${ }^{8-10}$.

Na literatura não há definição sobre a forma de tratamento das fraturas côndilares. Entretanto, único senso comum quanto à escolha de tratamento para essas fraturas é abordagem de maneira conservadora (método fechado) ou maneira cirúrgica (método aberto) ${ }^{11}$.

As fraturas de côndilo mandibular têm sido tratadas conservadoramente, utilizando a técnica de bloqueio maxilo-mandibular e fisioterapia para restabelecimento funcional ${ }^{10}$. Com advento dos sistemas de fixação interna rígida (placas e parafusos de titânio), houve a abertura de novas possibilidades para a indicação do tratamento cirúrgico. Entretanto, em virtude da biomecânica da articulação temporo-mandibular, da complexa anatomia local, da natureza da fratura, das peculiaridades de cada paciente e da diversidade de resultados com o mesmo tipo de tratamento, poucas áreas da cirurgia oral e maxilofacial têm gerado tanta controvérsia quanto a melhor forma de tratamento ${ }^{8,11,12}$.

Diversos fatores influenciam na escolha pela abordagem cirúrgica ou conservadora para tratamento das fraturas côndilares como, por exemplo: idade, comorbidade ao qual paciente seja portador, localização, tipo da fratura, grau de deslocamento, direção do segmento fraturado, presença de outras fraturas faciais associadas, presença de dentes, possibilidade de restabelecimento da oclusão dentária e hábitos nocivos ${ }^{6,13}$.

A abordagem conservadora (técnica fechada) consiste no reestabelecimento oclusal por meio da realização ou não de bloqueio maxilo-mandibular, por um período variando de 30 a 60 dias, fisioterapia elástica rigorosa e instituição de dieta líquida pastosa $^{2,10}$.

Já as indicações absolutas para adoção de abordagem aberta de fraturas côndilo são: deslocamento do côndilo para o interior da fossa craniana média, impossibilidade de obtenção de uma adequada oclusão pela técnica fechada, deslocamento extracapsular lateral do côndilo, presença de corpos estranhos dentro de a cápsula articular, obstrução mecânica impedindo a função da articulação têmporo-mandibular, fraturas expostas que requerem tratamento imediato ${ }^{11}$. Como todo procedimento cirúrgico, existem riscos de complicações pós-operatórias, dentre as mais comuns descritas na literatura, estão reabsorção côndilar, anquilose da articulação temporo-mandibular e má união; principalmente no tratamento cruento das fraturas altas e/ou intracapsulares ${ }^{9,14}$.

É de extrema importância a compreensão quanto à mobilização precoce da articulação temporomandibular; ser crucial para o adequado tratamento e restabelecimento da função articular ${ }^{8,9,15}$.

A adoção pelo tratamento aberto ou fechado estará baseada em alguns fatores que se correlacionam intimamente, tais como: experiência da equipe cirúrgica, nível e altura de deslocamento da fratura, referência oclusal e perfil do paciente, uma vez que na literatura não existe diferença estatisticamente de dados entre as formas de tratamento, que determine uma sobreposição de um tratamento sobre outro na esfera de melhores resultados clínicos $^{11,15}$. No serviço de Cirurgia e Traumatologia Bucomaxilofacial da Universidade Federal de Uberlândia o critério para adoção do tratamento cirúrgico das fraturas côndilares, baseia-se na viabilidade de fixação de uma ou duas placa de quatro furos.

No caso descrito optou-se pela abordagem não cirúrgica por um motivo principal, a fratura foi classificada como intracapsulares, o que torna a abordagem cruenta contraindicada. Em 18 meses de acompanhamento o paciente apresentou oclusão funcional restabelecida satisfatória e extinção do quadro álgico pré-auricular, o que remete resultado final satisfatório, corroborando com os dados presentes na literatura. Nos exames de imagem de dezoito meses de acompanhamento, notamos remodelação morfológica côndilar, dentro das expectativas considerando à natureza do trauma.

\section{CONSIDERAÇÕES FINAIS}

As fraturas de côndilo são comuns dentre as fraturas mandibulares, sendo os acidentes de trânsito e quedas os principais fatores etiológicos significativos.

A abordagem terapêutica deve considerar o tipo de fratura, as características clínicas, imaginológicas, localização anatômica e as peculiaridades de cada paciente para determinar a escolha da conduta terapêutica a ser adotada. É pertinente reforçar que o objetivo do tratamento de fraturas côndilares é a restauração da função mandibular, uma vez que a função mastigatória interfere diretamente na qualidade de vida do indivíduo, como mostram os casos clínicos relatados nesse estudo.

Mesmo com as distintas vertentes literárias quanto ao tratamento das fraturas côndilares é de ponto em comum que intervenção imediata e retorno precoce das atividades funcionais promovem um melhor prognóstico no tratamento de fraturas.

\section{REFERÊNCIAS}

01. Araújo A, Gabrielli MFR, Medeiros PJ. Aspectos atuais da cirurgia e traumatologia bucomaxilofacial. 1. ed. São Paulo: Editora Santos; 2007.

02. ManganelloLC, Silva AAF. Fraturas do côndilo mandibular: classificação e tratamento. Rev Bras Otorrinolaringol. 2002; 68(5) 249-255.

03. Souza LCM, Lucca MES. Fratura de mandíbula: análise de 282 pacientes. Rev Paul Odontol. 1992; 14(1): 2-4.

04. Araújo CFSN, Braga PLS, Ferreira JDB. Rev Cir Traumatol BucoMaxilo-Fac Camaragibe. 2013; 13(2):17-24.

05. Niedzielska IA, Tomczyk-Wzitek AM, Borowski BP. Fractures of the mandibular condylar processes- literature review. Cent Eur J Med. 2013; 8(2): 244-9. 
06. Smets LM, Van Damme PA, Stoelinga PJ. Non-surgical treatment of condylar fractures in adults: a retrospective analysis. J Cranio maxilla fac Surg. 2003; 31(3): 162-7.

07. Spiessl B, Schroll K. Internal fixation of the mandible: rigid internal fixation of fractures of the lower jaw. Reconstr Surg Traumatol. 1972; 13: $124-40$.

08. Silva J, Cauás M. Fratura de mandíbula decorrente de acidente automobilístico- Relato de caso. Odontologia. Clín Científ. 2004; 3(3): 199-208.

09. Walker RV. Condylar fractures: nonsurgical management. J Oral Maxillo fac Surg. 1994; 52(11): 1185-8.

10. Abdo Filho RCCA. Fratura bilateral de cabeça de mandíbula. Rev Saud e Pesq. 2011; 4(3): 449-54.
11. Riu G, Gamba U, Anghinoni M, Sesenna E. A comparison of open and closed treatment of condylar fractures: a change in philosophy. Int J Oral Maxilla Fac Surg. 2001; 30(5): 384-389.

12. Whaites E. Princípios de radiologia odontológica. 4. ed. Rio de janeiro: Elsevier; 2009. 408 p.

13. Andrade EF, Martins DMFS, Neto MS, Júnior CST, Pereira MD, Ferreira LM. Fraturas do côndilo mandibular: análise clínica retrospectiva das indicações e do tratamento. Rev Assoc Med Bras. 2003; 49(1): 54-9.

14. Abdo Filho RCCA et al. Fratura bilateral de cabeça de mandíbula. Rev Saud e Pesq. 2011; 4(3): 449-54.

15. Laskin DM. Management of condylar process fractures. Oral Maxillo fac Clin North Am. 2009; 21(2): 193-6.

\section{ABSTRACT}

Objective: This study aims to describe a clinical case of a patient with a condylar fracture treated in a bloodless manner with a follow - up of eighteen months. Case report: a young male patient suffering from a motorcycle accident with anterior open bite, limited mouth opening, bilaterally pain in the temporomandibular joints, absence of the condyles opening, limitation of lateral movements and mandibular protrusion. Imaging examinations were performed and diagnosed with a high condyle bilateral fracture. The conduct adopted was the installation of bimaxillary erich bar and maxillo-mandibular block for 21 days, posterior installation of elastics to guide occlusion, intensive physiotherapy and ambulatory follow-up monthly. Final considerations: even with the different literary aspects regarding the treatment of condylar fractures, the closed treatment is effective, and it is agreed that early diagnosis, immediate intervention and extemporaneous return of functional activities, aiming to promote a better prognosis in the treatment of fractures.

Keywords: Mandibular Condyle; Temporomandibular Joint; Vertical Dimension; Conservative Treatment.

\section{AUTOR PARA CORRESPONDÊNCIA}

Cristóvão Marcondes de Castro Rodrigues

Departamento de Cirurgia e Traumatologia

Bucomaxilofacial e Implantodontia

Endereço: Av. Pará, 1748 - Umuarama, Uberlândia - MG,

38405-320 Bloco 4T - Uberlândia Minas Gerais-MG.

Telefone: (34)999062400

E-mail: cristovao-marcondes@hotmail.com 